\title{
LA FORMULACIÓN DE PREGUNTAS EN EL AULA DE CLASE: UNA EVIDENCIA DE APRENDIZAJE SIGNIFICATIVO CRÍTICO
}

\section{The formulation of questions in the classroom: evidence of critical meaningful learning}

\author{
Sonia López ${ }^{1}$ • Eliane Angela Veit ${ }^{2}$. Ives Solano Araujo
}

Resumen: En este trabajo se muestran los principales resultados de un estudio que tuvo como propósito valorar la implementación de una propuesta didáctica constituida por diferentes actividades de modelación computacional haciendo uso del diagrama AVM - Adaptación de la V de Gowin a la Modelación Computacional -, así como su contribución en la habilidad adquirida por los estudiantes para formular preguntas en la clase de Física. El estudio fue realizado con un grupo de siete estudiantes de Física de la Universidad de Antioquia, Colombia; y estuvo fundamentado en la Teoría del Aprendizaje Significativo Crítico de Moreira - basada en las ideas de Postman y Weingartner de la enseñanza como una actividad subversiva- y en la modelación computacional con diagrama AVM. Los principales hallazgos develan que la implementación de la propuesta didáctica favoreció un progreso significativo en la habilidad de los estudiantes para formular preguntas de interés sobre la dinámica Newtoniana como campo de conocimiento.

Palabras clave: Enseñanza de la física. Aprendizaje significativo crítico. Modelación científica. Modelación computacional. Diagrama AVM.

\begin{abstract}
This paper presents the main results of a study aimed to assess the implementation of a didactic proposal made up of computational modeling activities, using the AVM diagram - an adaptation of Gowin's V to computational modeling-, and its impact on empowering students to formulate questions in physics class. The investigation was carried out with a group of seven students of physics from the Universidad de Antioquia, Colombia. The study is theoretically grounded in the principles of Moreira's Critical Meaningful Learning Theory -based on Postman and Weingartner's ideas of teaching as a subversive activity- and also in computational modeling using the AVM diagram. The main results reveal that the implementation of the didactic proposal had a positive impact in the development of students' ability to formulate questions about Newtonian dynamics as a field of knowledge.
\end{abstract}

Keywords: Physics teaching. Critical meaningful learning. Scientific modeling. Computational modeling. AVM diagram.

\footnotetext{
${ }^{1}$ Universidad de Antioquia, Facultad de Educación, Departamento de Enseñanza de las Ciencias y las Artes, Calle 67 \# 53-108, oficina 9-432, AA 1226, Medellín, Colombia. E-mail: slopez@ayura.udea.edu.co.

${ }^{2,3}$ Universidade Federal do Rio Grande do Sul (UFRGS), Instituto de Física, Porto Alegre, RS, Brasil.
} 
López, S.; Veit, E. A.; Araujo, I. S.

\section{Introducción}

La formulación de preguntas en el aula de clase ha sido tradicionalmente una tarea propia de los docentes, quienes - en muchas ocasiones - esperan que las respuestas de los estudiantes estén expresadas en los términos de sus discursos, negándoles así la posibilidad de cuestionamiento; de tal manera que los estudiantes formulan pocas preguntas en el aula de clase y, además las preguntas frecuentemente formuladas tienen un bajo nivel cognitivo (DILLON, 1988; OTERO; GRAESSER, 2001). Esto nos sugiere replantear esta actividad en el aula de clase, al asumir que la formulación de preguntas por parte de los estudiantes es una estrategia cognitiva que les permite lograr habilidades de orden superior. (ROSENSHINE; MEISTER; CHAPMAN, 1996).

La importancia de que los estudiantes desarrollen la capacidad de formular preguntas tiene un trasfondo epistemológico; de acuerdo con Bachelard (1982, p. 16), "para un espíritu científico todo conocimiento es una respuesta a una pregunta. Si no hubo pregunta, no puede haber conocimiento científico".

Enmarcados en la visión de Moreira (2005) en relación con la posibilidad de generar un aprendizaje significativo crítico en el aula de clase, nos enfocamos en el primer - y a nuestro modo de ver el más importante - postulado de la Teoría del Aprendizaje Significativo Crítico propuesta por este autor, que hace referencia al principio del cuestionamiento; es decir, al acto de enseñar/aprender preguntas en lugar de respuestas.

El favorecimiento de un aprendizaje significativo crítico en el aula de clase implica una estimulación del cuestionamiento por parte del estudiante; de manera que éste no sea un receptor pasivo del conocimiento transmitido por el profesor; y que por el contrario, asuma un papel activo en el proceso de aprendizaje (BOBBY et al., 2007; COLLINS; BROWN; NEWMAN, 1990; KING, 1994; SINGER, 1978); aprendiendo a formular de manera sistemática preguntas relevantes, apropiadas y sustantivas; y así generar en el aula de clase una permanente interacción estudiante-profesor y estudiante-estudiante.

Consideramos que el hecho de permitir a los estudiantes cuestionarse de un modo sistemático acerca de los diferentes fenómenos y hechos que les rodean, fomenta un aprendizaje significativo que les permite recurrir a un conocimiento previo de forma no arbitraria y no literal (AUSUBEL, 2002; MOREIRA, 2000); de tal manera que la calidad de las preguntas formuladas por los estudiantes tiene una estrecha relación con el conocimiento previo que poseen sobre un determinado tópico (NEBER; ANTON, 2008). Además, favorecer el cuestionamiento en el aula de clase a partir de la interacción social posibilita la negociación de significados, que a su vez implica un intercambio permanente de preguntas como fuente del conocimiento humano y como actividad fundamental de la ciencia; en la que el conocimiento no sea visto como "verdadero" y las respuestas como "correctas".

Asumiendo la modelación científica como una actividad propia de la ciencia, que puede entenderse como un proceso de construcción de modelos con el propósito de aprehender la realidad (BUNGE, 1972; GIERE, 1988; HALLOUN, 1996, 2004; HESTENES, 1995;) y de dar respuestas a preguntas formuladas sobre hechos reales o supuestos reales, en las que se fundamenta el conocimiento científico; consideramos factible incorporar en el aula de clase elementos propios del proceso de modelación científica, mediante actividades de modelación computacional y el uso del diagrama AVM (ARAUJO, 2005; ARAUJO; VEIT; MOREIRA, 
La formulación de preguntas en el aula ...

2012) y valorar su contribución en la habilidad adquirida por los estudiantes para formular preguntas de interés sobre situaciones físicas relativas al campo de la dinámica Newtoniana.

\section{Referentes conceptuales}

En coherencia con el interés de este estudio, el principal referente teórico propuesto para su orientación es la Teoría del Aprendizaje Significativo Crítico (TASC) de Moreira (2005), en particular lo referente al principio del cuestionamiento. El Aprendizaje Significativo Crítico es una teoría propuesta por Moreira (2005), basándose en las ideas de Postman y Weingartner (1969) expuestas en su libro La enseñanza como una actividad subversiva; y en la que se considera que el aprendizaje debe ser no sólo significativo, sino también subversivo o crítico; a partir de lo cual, Moreira (2005) propone nueve principios, ideas o estrategias facilitadoras del aprendizaje significativo crítico susceptibles de ser implementadas en el aula de clase. Estos principios son descritos a continuación de una manera muy sucinta.

- Principio 1. De la interacción social y del cuestionamiento. Enseñar/aprender preguntas en lugar de respuestas: el hecho de permitirle a los estudiantes cuestionarse de un modo sistemático acerca de los diferentes fenómenos y hechos que les rodean, fomenta un aprendizaje significativo que les permite recurrir a un conocimiento previo de forma no arbitraria y no literal.

- Principio 2. De la no centralización en el libro de texto. Del uso de documentos, artículos y otros materiales educativos. De la diversidad de materiales educativos: combatir la idea de que el libro de texto es el gran poseedor de conocimientos. El conocimiento es hoy documentado en diversas fuentes de información cada vez más accesibles; dentro de las cuales el libro de texto sigue siendo una de ellas, pero no la única.

- Principio 3. Del aprendiz como perceptor/representador: es preciso ver al estudiante no como un mero receptor de información; pues éste al recibir una nueva información la percibe y la representa mentalmente con base en sus percepciones previas. La información que el aprendiz recibe es percibida por éste y a partir de ella representa el mundo.

- Principio 4. Del conocimiento como lenguaje: el lenguaje está necesariamente implicado en cualquier intento humano de percibir la realidad. La comprensión de un área disciplinar implica comprender su lenguaje; es decir, sus palabras, signos, instrumentos y procedimientos. Aprender el lenguaje propio de un área de conocimiento es aprender otra manera de percibir y representar el mundo.

- Principio 5. De la conciencia semántica: el significado está en las personas y no en las palabras. Las personas atribuyen significado a las palabras con base en sus conocimientos previos, pero estos significados no son permanentes en el tiempo, cambian. La palabra es un símbolo que representa una cosa, que significa esa cosa, pero no es la cosa en sí. 
- Principio 6. Del aprendizaje por error: de acuerdo con este principio el hombre aprende corrigiendo sus errores, asumiendo que no hay verdades absolutas y que el conocimiento está en permanente cambio. Desde esta perspectiva, aprender críticamente implica buscar sistemáticamente el error considerándolo como algo natural en el proceso de aprendizaje.

- Principio 7. Del desaprendizaje: debido a que vivimos en un mundo en permanente cambio y transformación, nos encontraremos en algunos casos en los que conceptos aprendidos previamente se vuelven obsoletos, razón por la cual, el sujeto debe aprender a identificar cuáles de esos conocimientos son relevantes para dotar de significado los nuevos conocimientos.

- Principio 8. De la incertidumbre del conocimiento: en este principio se pone de relieve que el conocimiento no está fundamentado en verdades absolutas, que es incierto justamente porque depende de las preguntas que nos hacemos sobre el mundo y porque está expresado a través de definiciones que fueron inventadas con cierta finalidad.

- Principio 9. De la no utilización de la pizarra, de la participación activa del alumno, de la diversidad de estrategias de enseñanza: al igual que el libro de texto, la pizarra sigue siendo vista por algunos como portadora de respuestas ciertas, de verdades absolutas. Es preciso hacer énfasis en la necesidad de utilizar diversas estrategias instruccionales o didácticas que posibiliten la participación activa de los estudiantes; lo que sin duda alguna facilita un aprendizaje significativo crítico a partir del intercambio de significados entre éstos y el profesor como mediador.

La Teoría del Aprendizaje Significativo Crítico es una teoría actual y poco difundida; y aunque los nueve principios en los que esta teoría se resume pueden ser perfectamente implementados en el aula de clase, en este trabajo presentamos de manera específica los resultados de la implementación del principio 1 de la TASC referido al cuestionamiento; por considerar que a partir de este principio es ampliamente factible abordar elementos propios de la modelación científica a partir de actividades de modelación computacional y del uso del diagrama $\mathrm{AVM}^{4}$, tomando como punto de partida el hecho de que el conocimiento está fundamentado en las preguntas que formulamos acerca del mundo que nos rodea.

La actividad de formulación de preguntas por parte de los estudiantes es apoyada también por la Modelación Computacional con diagrama AVM. Como es bien sabido, la modelación computacional se ha convertido en una potencial herramienta para la enseñanza de las ciencias, ya que permite dar cuenta de un fenómeno estudiado desde distintos puntos de vista de modo más simple y directo que la experimentación convencional en un laboratorio, convirtiéndose además en un valioso complemento para el trabajo experimental. Asimismo, la modelación computacional permite al estudiante construir una idea, representación, imagen o modelo mental a partir de imágenes externas, necesarias para la comprensión del mundo físico. No obstante, la incorporación de las actividades de modelación computacional en el aula

\footnotetext{
${ }^{4}$ Las actividades de modelación computacional con uso del diagrama AVM, pueden verse en: http://dspace.ubu.es:8080/tesis/handle/10259/177
} 
de clase necesariamente debe estar orientada por una estrategia que acompañe dicho procedimiento y permita visualizar el proceso de construcción de conocimiento a partir de modelos.

Desde esta perspectiva, se propone el diagrama AVM - desde sus dominios conceptual y metodológico - como una herramienta que permite explorar y/o construir modelos computacionales, abordando todos y cada uno de los componentes necesarios para su comprensión; y que de acuerdo con Araujo, Veit y Moreira (2007), tiene como objetivo primordial fomentar la reflexión crítica por parte de los alumnos, sobre los modelos físicos abordados, contribuyendo así a un aprendizaje significativo crítico.

El diagrama AVM es una estrategia de enseñanza-aprendizaje que apoya a los estudiantes en el proceso de interacción con actividades de modelación computacional y que permite al profesor o investigador valorar la contribución de estas actividades al aprendizaje. Así, Araujo (2005) presenta el diagrama AVM como un instrumento heurístico elaborado a partir de la adaptación de la $\mathrm{V}$ de Gowin para actividades que involucran el uso de modelación computacional. El formato $\mathrm{V}$ es adoptado por evidenciar la interacción entre los dos dominios indispensables para la construcción de un modelo computacional dirigido a los procesos de enseñanza y aprendizaje de la Física: el dominio teórico relacionado con la concepción del modelo computacional y el dominio metodológico asociado a la implementación y/o exploración de este modelo.

La Figura 1 muestra cada uno de los componentes de la versión más reciente del diagrama $\mathrm{AVM}^{5}$. Es importante resaltar que hay una permanente interacción entre los dos lados del diagrama, de modo que todo lo que es hecho en el lado metodológico está guiado por los componentes del lado teórico o conceptual, con el objetivo de construir/analizar el modelo y dar respuesta a las preguntas foco.

Para la implementación del diagrama AVM en actividades de modelación computacional, Araujo, Veit y Moreira (2012) proponen cuatro modos básicos de uso del diagrama AVM:

1) Modo exploratorio dirigido: en el diagrama AVM, el objetivo general, las preguntas foco y la situación foco son definidas por el profesor y una simulación computacional es presentada. La elaboración reflexiva de la $\mathrm{V}$ servirá como una guía para la exploración del modelo de manera que se respondan las preguntas planteadas.

2) Modo exploratorio abierto: es presentada una simulación computacional y se pide al alumno que a través del diagrama AVM, explore de forma reflexiva el modelo, dando especial atención a la formulación de las preguntas foco.

3) Modo expresivo dirigido: en este caso el objetivo general, las preguntas foco y la situación foco son aportados previamente por el profesor, dejando a cargo del alumno la elaboración del resto de la $\mathrm{V}$ y la construcción del modelo computacional correspondiente.

\footnotetext{
${ }^{5}$ En este estudio fue utilizada la versión original del diagrama AVM (ARAUJO, 2005) que difiere de la versión más reciente en aspectos como: a) no se consideraba el objetivo general, sino el fenómeno de interés, b) se incluía el componente relativo a la filosofía como primer elemento del dominio conceptual, y c) en el campo de variables, parámetros, constantes y sus representaciones, eran tenidos en cuenta solamente los dos primeros elementos; es decir, variables y parámetros.
} 
López, S.; Veit, E. A.; Araujo, I. S.

4) Modo expresivo abierto: son propuestas actividades en que el alumno debe construir el modelo computacional a partir de la elaboración reflexiva del diagrama AVM, definiendo él mismo el objetivo general, las preguntas foco y la situación foco que guiarán su trabajo. Este modo de uso del diagrama AVM puede guiar al profesor y a los alumnos en la construcción de sus propios modelos.

En el ámbito de este trabajo, fueron implementados dos de estos modos básicos de uso del diagrama AVM; el modo exploratorio abierto y el modo expresivo abierto, en el proceso de interacción y construcción, respectivamente, de modelos computacionales ${ }^{6}$.

Figura 1. Diagrama AVM (Adaptación de la V de Gowin para la modelación computacional).

DOMINIO CONCEPTUAL

Teorías, principios, conpcetos y leyes

Referentes reales

idealizaciones y aproximaciones

Variables, parámetros, constantes y sus representaciones

Relaciones matemáticas y/o proposicionales

Resultados conocidos

Predicciones
DOMINIO METODOLÓGICO

Posibles generalizaciones y expansiones del modelo

Respuestas a las preguntas foco

Validación del modelo

Categorización de la actividad computacional

Elementos interactivos

Forma de presentación de los registros

Registros

Situación foco

Fuente: Araujo, Veit y Moreira (2012).

\footnotetext{
${ }^{6}$ Los modelos computacionales fueron construidos con el software Modellus 2.5; un software gratuito particularmente apropiado para el desarrollo de modelos computacionales referentes al campo de la dinámica Newtoniana. Un software de fácil manejo y ejecutable en la mayoría de los computadores; su entorno gráfico es amigable y compatible con el diseñado por Microsoft y también funciona en un ambiente Linux que disponga de emulador de Windows. Inclusive, la versión más actual del programa es multiplataforma, pudiendo ser ejecutado en cualquier sistema operativo que disponga de una máquina Java actualizada.
} 
La formulación de preguntas en el aula ...

\section{Metodología}

En este estudio fue adoptada una metodología cualitativa del tipo estudio de casos. En la perspectiva de la investigación cualitativa-interpretativa, se privilegia el estudio de casos (ARNAL; DEL RINCÓN; LATORRE, 1992), ya que éste permite profundizar y comprender lo que pasa con una persona, un grupo, una institución o una comunidad; pues tal y como lo afirma Stake (1998), el estudio de casos es el estudio de la particularidad y de la complejidad de un caso singular para llegar a comprender su actividad en circunstancias importantes. Por las características de esta investigación se implementa el estudio de casos interpretativo en los términos planteados por Pérez-Serrano (1998), atendiendo a razones como la definición previa de la orientación teórica que guía la investigación y por el predominio de la generación de categorías conceptuales que aportan a la comprensión del fenómeno.

Este estudio se llevó a cabo con un grupo de siete estudiantes que cursaban la asignatura Didáctica para Físicos del programa de Física de la Facultad de Ciencias Exactas y Naturales de la Universidad de Antioquia, Colombia. Al momento de la intervención todos los estudiantes habían cursado todas las asignaturas de Física Clásica, e inclusive algunos de ellos habían cursado algunas asignaturas de Física Moderna. Este estudio se realizó durante el primer semestre de 2009 con una intensidad de cuatro horas semanales divididas en dos sesiones (dos horas cada una). Sin tener en cuenta el tiempo dedicado a la aplicación de instrumentos para la recolección de la información inicial y final de este estudio, las actividades de la propuesta didáctica relativas a la modelación computacional se llevaron a cabo durante quince sesiones. Algunas de estas actividades fueron realizadas de manera individual y otras en pequeños grupos de dos o tres estudiantes, siempre bajo la orientación del profesor investigador.

Solamente cinco de las actividades de la propuesta didáctica realizadas durante nueve sesiones hacían referencia al principio del cuestionamiento abordado en este estudio. En el Cuadro 1 se muestran específicamente las actividades e instrumentos para la recolección de información, los objetivos y el tiempo de duración de cada una de estas sesiones.

El principio 1 de la TASC atendido en este estudio se refiere a la capacidad desarrollada por los estudiantes para formular preguntas relevantes, apropiadas y sustantivas de manera sistemática; considerándose ésta una evidencia de aprendizaje significativo crítico. Las actividades de la propuesta didáctica descritas en el Cuadro 1 permiten conocer el progreso y evolución de los estudiantes en la habilidad para formular preguntas, propiciados por el uso de la modelación computacional con diagrama AVM. Tal evolución es valorada a partir de una escala ordinal, estableciendo tres niveles -bajo, medio, alto- en la capacidad de formular preguntas, como se muestra en el Cuadro 2.

\section{Presentación y discusión de resultados}

Como ya fue mencionado, este estudio se realizó con un grupo de siete estudiantes, constituyéndose cada uno de ellos en un caso de análisis. De los siete estudiantes, cinco eran hombres y dos mujeres, cuyas edades oscilaban entre los 21 y 35 años; y se encontraban cursando entre el séptimo y noveno nivel del programa. Se presenta la valoración del progreso de tres de los estudiantes participantes en el estudio -estudiantes 4, 5 y 7-, por considerar que 
López, S.; Veit, E. A.; Araujo, I. S.

Cuadro 1. Actividades e instrumentos de la propuesta didáctica para la recolección de información relacionada con el principio del cuestionamiento.

\begin{tabular}{|c|c|c|}
\hline $\begin{array}{l}\text { Actividades e Instrumentos } \\
\text { para la recolección de información }\end{array}$ & Objetivo & Duración \\
\hline $\begin{array}{l}\text { 1. Lectura crítica y discusión del artículo "A } \\
\text { aerodinâmica da bola de futebol" de Aguiar y Rubini } \\
\text { (2004); en el cual se realizaba el análisis de un } \\
\text { fenómeno físico cotidiano como es el lanzamiento } \\
\text { de un balón de fútbol, a partir del uso de } \\
\text { herramientas computacionales. En esta actividad } \\
\text { los estudiantes identificaron elementos como: } \\
\text { fenómeno de interés, teorías, conceptos, } \\
\text { idealizaciones; y se les incitó a formular preguntas } \\
\text { de interés para el análisis de dicho fenómeno. }\end{array}$ & $\begin{array}{l}\text { Familiarizar a los estudiantes con } \\
\text { elementos del proceso de modelación } \\
\text { científica, por medio de la identificación } \\
\text { de algunos de sus principales } \\
\text { elementos y de la formulación de } \\
\text { preguntas. }\end{array}$ & $\begin{array}{c}4 \text { horas } \\
\text { (2 sesiones) }\end{array}$ \\
\hline $\begin{array}{l}\text { 2. Formulación de preguntas por parte de los } \\
\text { estudiantes a partir de situaciones problema } \\
\text { referentes a la dinámica Newtoniana. }\end{array}$ & $\begin{array}{l}\text { Promover la formulación de preguntas } \\
\text { de interés para situaciones problema } \\
\text { propuestas en el marco de la dinámica } \\
\text { Newtoniana. }\end{array}$ & 2 horas \\
\hline $\begin{array}{l}\text { 3. Modelación computacional de libre exploración: } \\
\text { los estudiantes formularon preguntas susceptibles } \\
\text { de ser respondidas a partir de la interacción con los } \\
\text { modelos computacionales aportados por el profesor. }\end{array}$ & $\begin{array}{l}\text { Posibilitar la formulación de preguntas } \\
\text { a partir de la exploración e interacción } \\
\text { con modelos computacionales. }\end{array}$ & $\begin{array}{c}4 \text { horas } \\
\text { (2 sesiones) }\end{array}$ \\
\hline $\begin{array}{l}\text { 7. Modelación computacional de tipo exploratorio } \\
\text { abierto con diagrama AVM: el diagrama debía ser } \\
\text { completamente diligenciado por los estudiantes, } \\
\text { prestando especial atención a la formulación de las } \\
\text { preguntas foco; a partir de la interacción con } \\
\text { modelos computacionales aportados por el profesor. }\end{array}$ & $\begin{array}{l}\text { Propiciar en los estudiantes la } \\
\text { capacidad para formular preguntas a } \\
\text { partir de las actividades } \\
\text { computacionales. }\end{array}$ & $\begin{array}{c}4 \text { horas } \\
\text { (2 sesiones) }\end{array}$ \\
\hline $\begin{array}{l}\text { 9. Modelación computacional de tipo expresivo } \\
\text { abierto con diagrama AVM: los estudiantes debían } \\
\text { construir los modelos computacionales y su } \\
\text { correspondiente diagrama AVM, a partir de las } \\
\text { situaciones problema propuestas. }\end{array}$ & $\begin{array}{l}\text { Incentivar en los estudiantes la } \\
\text { habilidad para formular preguntas y } \\
\text { diseñar modelos computacionales. }\end{array}$ & $\begin{array}{c}4 \text { horas } \\
(2 \text { sesiones) }\end{array}$ \\
\hline
\end{tabular}

Fuente: Elaborado por los autores.

son los casos más representativos de éste. Un criterio fundamental para la elección de estos tres casos fue el rendimiento de los estudiantes en las diferentes actividades de la propuesta didáctica implementada; de donde se elige un estudiante con desempeño bajo (Estudiante 7), un estudiante con desempeño medio (Estudiante 4) y un estudiante con desempeño alto (Estudiante 5). Otro criterio que posibilitó la elección de estos tres casos es el hecho de que estos estudiantes nunca coincidieron en un mismo grupo de trabajo -con excepción de los estudiantes 4 y 7 que trabajaron conjuntamente en la actividad 9 de la propuesta didáctica-.

En los Cuadros 3, 4 y 5 se describen las actividades en las que cada estudiante participó, las preguntas formuladas por éste y el nivel de valoración de las preguntas de acuerdo con el Cuadro 2. 
La formulación de preguntas en el aula ...

Cuadro 2. Escala cualitativa de valoración para la capacidad de formular preguntas.

\begin{tabular}{|c|l|}
\hline $\begin{array}{c}\text { Niveles en la capacidad } \\
\text { deformular preguntas }\end{array}$ & \multicolumn{1}{c|}{ Características de las preguntas } \\
\hline Bajo & $\begin{array}{l}\text { Se hace referencia a preguntas muy limitadas; preguntas que indagan por } \\
\text { respuestas numéricas, respuestas del tipo sí o no, o preguntas que buscan como } \\
\text { respuesta una ecuación o una palabra completamente inducida por la pregunta; } \\
\text { así, como preguntas que indagan por aspectos muy generales que pueden ser } \\
\text { subyacentes al fenómeno estudiado, pero que no posibilitan su comprensión. }\end{array}$ \\
\hline Medio & $\begin{array}{l}\text { Aquellas preguntas cuyas respuestas requieren el uso de algún o algunos } \\
\text { conceptos en la descripción de situaciones o fenómenos, sin que se establezca } \\
\text { necesariamente una relación entre éstos. Asimismo, pueden considerarse en este } \\
\text { nivel, preguntas que pueden generar cierto interés pero que poco aportan a la } \\
\text { comprensión del fenómeno en cuestión. }\end{array}$ \\
\hline Alto & $\begin{array}{l}\text { Aquellas preguntas que incitan al estudiante a la conceptualización, preguntas que } \\
\text { requieren una comprensión de los conceptos involucrados en el análisis de un } \\
\text { fenómeno y que posibilitan el establecimiento de relaciones claras y coherentes } \\
\text { entre los conceptos que explican dicho fenómeno. }\end{array}$ \\
\hline
\end{tabular}

Fuente: Elaborado por los autores.

A partir de las actividades 1 y 2 de la propuesta didáctica puede verse que en las preguntas formuladas por el estudiante 4 predominan aquellas que requieren respuestas del tipo sí o no, preguntas que no brindan la posibilidad de una conceptualización por parte del estudiante a la hora de intentar comprender el fenómeno estudiado. La actividad 3 denominada modelación computacional de libre exploración, no fue realizada por el estudiante 4.

En la actividad 7 el estudiante 4 formula preguntas en relación con los modelos computacionales fricción y paracaídas. A partir de estas preguntas puede decirse que este estudiante logró un cierto avance en su capacidad para formular preguntas, si se compara con las formuladas en actividades anteriores. Sin embargo, las preguntas todavía no generan una buena conceptualización en relación con los fenómenos de interés; es decir, no propician el establecimiento de relaciones entre los principales conceptos involucrados en las situaciones problema planteadas.

En la actividad 9 referida a la modelación computacional de modo expresivo abierto, el estudiante 4 diseñó un modelo computacional para el oscilador armónico amortiguado. Las preguntas formuladas para este modelo son preguntas válidas para el análisis de la situación física planteada; sin embargo, no contribuyen en su totalidad a la interacción con el modelo computacional y por supuesto a la comprensión del sistema físico estudiado; a partir de lo cual puede afirmarse que la capacidad del estudiante 4 para formular preguntas de interés acerca de fenómenos relacionados con la dinámica Newtoniana se encuentra en un nivel medio.

Aunque este estudiante sigue mostrando algunas dificultades en cuanto a la formulación de preguntas, que hacen presumir que aún debe progresar mucho para adquirir tal habilidad; es importante reconocer que se logró un cierto avance en esta capacidad formulando preguntas cada vez más pertinentes y más reflexivas en torno a fenómenos de la dinámica Newtoniana. 
López, S.; Veit, E. A.; Araujo, I. S.

Cuadro 3. Progreso del estudiante 4 en el proceso de formulación de preguntas.

\begin{tabular}{|c|c|c|}
\hline Actividades & Preguntas formuladas & Valoración \\
\hline $\begin{array}{l}\text { 1. Lectura crítica y discusión } \\
\text { del artículo "A aerodinâmica } \\
\text { da bola de futebol" }\end{array}$ & $\begin{array}{l}\text { “Los efectos de arrastre generados por el aire son los } \\
\text { causantes del movimiento visto?” } \\
\text { ¿El momento angular del balón y los efectos de arrastre } \\
\text { describen el movimiento?” }\end{array}$ & Bajo \\
\hline $\begin{array}{l}\text { 2. Formulación de preguntas } \\
\text { por parte de los estudiantes a } \\
\text { partir de situaciones problema } \\
\text { referentes a la dinámica } \\
\text { Newtoniana. }\end{array}$ & $\begin{array}{l}\text { Un jugador de basketball lanza un balón en dirección a la cesta } \\
\text { ¿Cuál es la manera más eficiente de lanzar un objeto que } \\
\text { describa este movimiento?" } \\
\text { ¿Qué tipo de trayectoria describe el objeto?" } \\
\text { Objeto sujeto a un hilo oscilando en un plano vertical } \\
\text { ¿El movimiento es periódico?” } \\
\text { ¿De qué depende el período?" } \\
\text { ¿Cómo varía la velocidad angular?" } \\
\text { Un cuerpo oscila unido a un resorte en posición vertical y } \\
\text { sumergido en un líquido viscoso } \\
\text { ¿La frecuencia de oscilación de este sistema es diferente del } \\
\text { péndulo en el vació?" } \\
\text { Un niño en un columpio es empujado por su padre } \\
\text { “¿El movimiento puede ser periódico?" }\end{array}$ & Bajo \\
\hline $\begin{array}{l}\text { 7. Modelación computacional } \\
\text { de tipo exploratorio abierto } \\
\text { con diagrama AVM }\end{array}$ & $\begin{array}{l}\text { Modelo computacional: fricción } \\
\text { ¿Cómo varía la magnitud de la fuerza aplicada durante la } \\
\text { evolución temporal del modelo?" } \\
\text { ¿Por qué si aplico una fuerza el bloque se mueve con } \\
\text { velocidad constante?” } \\
\text { Modelo computacional: Fenómeno del paracaídas } \\
\text { “Cuál es la causa de que el paracaídas se abra?” } \\
\text { ¿Cómo se evidencia la tercera ley de Newton?” }\end{array}$ & Bajo-medio \\
\hline $\begin{array}{l}\text { 9. Modelación computacional } \\
\text { de tipo expresivo abierto con } \\
\text { diagrama AVM }\end{array}$ & $\begin{array}{l}\text { Un cuerpo oscila unido a un resorte en posición vertical y } \\
\text { sumergido en un líquido viscoso } \\
\text { “Cómo se describe el comportamiento del sistema?" } \\
\text { ¿Cómo es el movimiento del sistema si se desprecia la } \\
\text { constante de amortiguamiento?" }\end{array}$ & Medio \\
\hline
\end{tabular}

Fuente: Elaborado por los autores.

Es importante destacar que este estudiante estuvo particularmente interesado en las diferentes actividades de modelación computacional, mostrando una muy buena predisposición para el desarrollo de estas y llevándolas a cabo de manera muy satisfactoria; y aunque manifestó su inclinación por el trabajo individual, accedió fácilmente a trabajar en grupo en las actividades del curso que así lo requerían.

A partir del Cuadro 4 en el que son mostradas las diferentes preguntas formuladas por el estudiante 5, puede observarse de modo particular que en la actividad 3 algunas de las preguntas que formula este estudiante a partir de la observación e interacción libre con los modelos computacionales (por ejemplo en el modelo referido al fenómeno del paracaídas), aunque no son las más representativas y explicativas del fenómeno, de alguna manera indagan por la relación entre conceptos.

De acuerdo con las preguntas foco formuladas por el estudiante 5 en la actividad 7 referida al análisis de los modelos de fricción y paracaídas, puede afirmarse que se dieron 
La formulación de preguntas en el aula ...

Cuadro 4. Progreso del estudiante 5 en el proceso de formulación de preguntas.

\begin{tabular}{|c|c|c|}
\hline Actividades & Preguntas formuladas & Valoración \\
\hline $\begin{array}{l}\text { 1. Lectura crítica y } \\
\text { discusión del artículo "A } \\
\text { aerodinâmica da bola de } \\
\text { futebol" }\end{array}$ & $\begin{array}{l}\text { “Cómo se modifica la trayectoria de un cuerpo esférico teniendo en } \\
\text { cuenta la crisis de arrastre y el efecto Magnus?” } \\
\text { ¿Cómo se comporta el fluido en las inmediaciones del cuerpo } \\
\text { esférico?” }\end{array}$ & Medio \\
\hline $\begin{array}{l}\text { 2. Formulación de } \\
\text { preguntas por parte de } \\
\text { los estudiantes a partir } \\
\text { de situaciones problema } \\
\text { referentes a la dinámica } \\
\text { Newtoniana. }\end{array}$ & $\begin{array}{l}\text { Un jugador de basketball lanza un balón en dirección a la cesta } \\
\text { ¿Qué trayectoria describe el proyectil y qué fuerzas la } \\
\text { determinan?” } \\
\text { ¿Para qué ángulo se alcanza una altura máxima y para qué otro } \\
\text { ángulo se alcanza una distancia horizontal máxima?” } \\
\text { Objeto sujeto a un hilo oscilando en un plano vertical } \\
\text { ¿Depende la amplitud del ángulo de inclinación?” } \\
\text { ¿Qué fuerzas actúan sobre el péndulo?” } \\
\text { Varilla sostenida de un pivote y oscilando en un plano vertical } \\
\text { ¿Cuál es la ecuación de movimiento?” } \\
\text { ¿Cuál es el período de las oscilaciones?” } \\
\text { Un cuerpo oscila unido a un resorte en posición vertical y sumergido } \\
\text { en un líquido viscoso } \\
\text { ¿cómo se halla la fuerza de fricción?” } \\
\text { Un niño en un columpio es empujado por su padre } \\
\text { ¿Qué fuerzas son responsables de las oscilaciones?” } \\
\text { ¿Qué parámetros influyen en la frecuencia de oscilación?” }\end{array}$ & Bajo \\
\hline $\begin{array}{l}\text { 3. Modelación } \\
\text { computacional de libre } \\
\text { exploración }\end{array}$ & $\begin{array}{l}\text { Modelo computacional: Tiro parabólico } \\
\text { "Para una velocidad inicial fija ¿ a qué ángulo se obtiene un alcance } \\
\text { horizontal máximo?" } \\
\text { ¿Cuáles son los vectores dibujados sobre el balón?" } \\
\text { Modelo computacional: Relación fuerza-aceleración } \\
\text { "Cuando la fuerza es negativa ¿cómo es el movimiento de la } \\
\text { masa?” } \\
\text { “Qué sucede con la velocidad a medida que pasa el tiempo?” } \\
\text { Modelo computacional: Fenómeno del paracaídas } \\
\text { ¿Qué sucede con la velocidad cuando el paracaídas es abierto?” } \\
\text { ¿Cómo varía la velocidad con el incremento de m y g?” }\end{array}$ & Medio \\
\hline $\begin{array}{l}\text { 7. Modelación } \\
\text { computacional de tipo } \\
\text { exploratorio abierto con } \\
\text { diagrama AVM }\end{array}$ & $\begin{array}{l}\text { Modelo computacional: fricción } \\
\text { ¿En qué instante comienza el bloque a moverse?” } \\
\text { ¿Qué sucede con el coeficiente de fricción cuando el bloque } \\
\text { empieza a moverse?” } \\
\text { ¿Qué sucede si } \mu c>\mu e \text { y si } \mu c<\mu e ? ” \\
\text { Modelo computacional: Fenómeno del paracaídas } \\
\text { ¿Qué efecto tiene la resistencia del aire sobre el paracaidista antes } \\
\text { de abrir el paracaídas y luego de abrirlo?” } \\
\text { ¿Qué sucede si el coeficiente de resistencia del aire es muy } \\
\text { grande y hay una velocidad inicial?” }\end{array}$ & Medio-alto \\
\hline $\begin{array}{l}\text { 9. Modelación } \\
\text { computacional de tipo } \\
\text { expresivo abierto con } \\
\text { diagrama AVM }\end{array}$ & $\begin{array}{l}\text { Un cuerpo oscila unido a un resorte en posición vertical y sumergido } \\
\text { en un líquido viscoso; y un niño en un columpio es empujado por su } \\
\text { padre } \\
\text { ¿Cuándo se presenta el fenómeno de resonancia?” } \\
\text { ¿Qué sucede si la constante de amortiguación es muy grande?” } \\
\text { ¿Cómo influye la masa de la partícula en el fenómeno de } \\
\text { resonancia?” }\end{array}$ & Medio \\
\hline
\end{tabular}

Fuente: Elaborado por los autores. 
logros significativos en relación con las preguntas formuladas en actividades previas; pues la mayoría de estas preguntas inducen al estudiante a realizar un análisis reflexivo del fenómeno de interés y a establecer relaciones entre conceptos para aportar las explicaciones requeridas. En relación con la actividad 9 concerniente a la modelación computacional expresiva abierta, el estudiante 5 construyó un modelo computacional en el que podía estudiarse tanto el oscilador armónico amortiguado como el oscilador forzado. No obstante, aunque las preguntas formuladas tienen cierto interés para el análisis de los sistemas físicos planteados, éstas son insuficientes para propiciar la comprensión del modelo computacional que representa dichos sistemas.

El estudiante 5 ha mostrado un alto rendimiento académico durante su carrera, y en este curso no fue la excepción. Demostró gran disposición para realizar las diversas actividades que hacían parte de la propuesta didáctica, así como para el trabajo en grupo.

A partir del análisis de la información recolectada para el estudiante 5 se encuentran interesantes evidencias de una mayor profundización de los conceptos relativos al campo de la dinámica Newtoniana. Esta afirmación puede sustentarse a partir del seguimiento realizado a este estudiante en las diferentes actividades relativas a la formulación de preguntas, donde de alguna manera se mantiene su habilidad para formular preguntas y en algunos casos, dicha habilidad es más notoria, sobre todo en las actividades finales de la intervención.

En relación con el estudiante 7, en el Cuadro 5 pueden observarse las preguntas formuladas por éste para las diferentes actividades de la propuesta didáctica; y al respecto, es importante resaltar que en la actividad 1 este estudiante hizo la lectura del artículo de manera individual y además envió sus respuestas escritas; pues no asistió a la sesión de clase en la que se realizó la discusión del artículo. Las preguntas formuladas por el estudiante 7 en esta actividad hacen referencia a las preguntas que estaban explícitamente formuladas por los autores del artículo de investigación analizado, por lo que no es posible hacer una valoración de sus preguntas.

Las preguntas que formula el estudiante 7 para la actividad 2 poco aportan a la comprensión de las situaciones físicas planteadas, predominando las preguntas que generan respuestas del tipo sí o no. Asimismo, las preguntas formuladas en la actividad 3, en general, son preguntas que poco interés pueden generar en los estudiantes, contribuyendo poco a la comprensión de los modelos computacionales explorados.

En la actividad 7 relacionada con la modelación computacional de modo exploratorio abierto, el estudiante 7 formula una serie de preguntas para el análisis de los modelos de fricción y paracaídas; y aunque a partir de esta actividad puede decirse que se nota un avance en relación con las preguntas formuladas en actividades anteriores, sus preguntas siguen siendo conceptualmente pobres, en cuanto no indagan por relaciones importantes entre los conceptos involucrados en los fenómenos analizados. La actividad 9 relacionada con la modelación computacional de modo expresivo abierto no se relaciona para el estudiante 7, ya que esta fue desarrollada por éste en conjunto con el estudiante 4 - y presentada en el Cuadro de este estudiante-, logrando un nivel medio en la formulación de preguntas, que resultaron como producto del consenso y negociación entre ambos.

El estudiante 7 se mostró comprometido con las diferentes actividades que hicieron parte de la intervención; sin embargo, la poca familiaridad que tenía con las actividades computacionales y sus grandes dificultades conceptuales en torno a la temática que estaba siendo 
La formulación de preguntas en el aula ...

Cuadro 5. Progreso del estudiante 7 en el proceso de formulación de preguntas.

\begin{tabular}{|c|c|c|}
\hline Actividades & Preguntas formuladas & Valoración \\
\hline $\begin{array}{l}\text { 1. Lectura crítica y } \\
\text { discusión del artículo "A } \\
\text { aerodinâmica da bola de } \\
\text { futebol" }\end{array}$ & $\begin{array}{l}\text { “Por qué causa no se hizo el gol?” } \\
\text { ¿Qué otros factores pudieron influir a la hora de patear el balón?” }\end{array}$ & 7 \\
\hline $\begin{array}{l}\text { 2. Formulación de } \\
\text { preguntas por parte de } \\
\text { los estudiantes a partir } \\
\text { de situaciones problema } \\
\text { referentes a la dinámica } \\
\text { Newtoniana. }\end{array}$ & $\begin{array}{l}\text { Un jugador de basketball lanza un balón en dirección a la cesta } \\
\text { “Considérese un jugador de basketball que lanza un balón, ¿qué } \\
\text { factores externos influyen para la trayectoria?” } \\
\text { ¿En qué dirección se presenta aceleración?” } \\
\text { Objeto sujeto a un hilo oscilando en un plano vertical } \\
\text { “ ¿Puede ser la tensión menor al peso del objeto?” } \\
\text { ¿Qué tipo de mecánica puede ser utilizada para conocer la } \\
\text { velocidad?” } \\
\text { Varilla sostenida de un pivote y oscilando en un plano Vertical } \\
\text { ¿Qué tipo de fuerza realiza torque?” } \\
\text { ¿Es armónico simple este movimiento?” } \\
\text { Un cuerpo oscila unido a un resorte en posición vertical y sumergido } \\
\text { en un líquido viscoso } \\
\text { “Considérese una masa atada a un resorte, ¿en qué punto es } \\
\text { máxima la energía potencial elástica?” } \\
\text { Un niño en un columpio es empujado por su padre ¿Puede la fuerza } \\
\text { realizar un movimiento armónico simple? }\end{array}$ & Bajo \\
\hline $\begin{array}{l}\text { 3. Modelación } \\
\text { computacional de libre } \\
\text { exploración }\end{array}$ & $\begin{array}{l}\text { Modelo computacional: Tiro parabólico } \\
\text { ¿Cuál es el ángulo de mayor alcance?” } \\
\text { ¿Existe aceleración en la dirección horizontal?" } \\
\text { Modelo computacional: Relación fuerza-aceleración } \\
\text { ¿Cuál es la ecuación que relaciona la fuerza con la aceleración?" } \\
\text { ¿Cómo varía la velocidad?” } \\
\text { Modelo computacional: Fenómeno del paracaídas } \\
\text { “Cómo se mueve el paracaidista si no hay resistencia?” } \\
\text { ¿Es posible que el paracaidista nunca caiga?” }\end{array}$ & Bajo \\
\hline $\begin{array}{l}\text { 7. Modelación } \\
\text { computacional de tipo } \\
\text { exploratorio abierto con } \\
\text { diagrama AVM }\end{array}$ & $\begin{array}{l}\text { Modelo computacional: fricción } \\
\text { ¿Qué pasa con la fuerza externa antes y después del } \\
\text { movimiento?” } \\
\text { ¿Qué sucede con el coeficiente de fricción en función del tiempo } \\
\text { antes y después del movimiento?” } \\
\text { ¿Existe un coeficiente de fricción para el que el movimiento sea } \\
\text { inminente?” } \\
\text { Modelo computacional: Fenómeno del paracaídas } \\
\text { ¿Cómo se comporta la velocidad del paracaidista durante su } \\
\text { movimiento considerando la resistencia del aire?” } \\
\text { ¿Si se varía el coeficiente asociado a la resistencia del aire, cómo } \\
\text { se ve afectada la velocidad del paracaidista?” }\end{array}$ & Bajo-medio \\
\hline
\end{tabular}

Fuente: Elaborado por los autores.

\footnotetext{
${ }^{7}$ El estudiante 7 describe las preguntas propuestas por los autores del artículo, por lo que no se hace una valoración de éstas.
} 
López, S.; Veit, E. A.; Araujo, I. S.

abordada, poco le permitieron contribuir al desarrollo de las actividades de modelación computacional; valorando el trabajo en grupo en términos de que su compañero de trabajo asumiera el manejo del programa computacional que él no estaba en capacidad de manejar. Esta actitud se evidenció particularmente en las actividades de modelación computacional de modo expresivo abierto.

A partir del análisis de los resultados obtenidos para el estudiante 7, es posible afirmar que éste obtuvo un cierto progreso en la habilidad para formular preguntas; sin embargo, éste no fue muy notorio, manteniéndose a lo largo de la intervención entre el nivel bajo y medio en su capacidad para formular preguntas sobre fenómenos de la dinámica Newtoniana.

Los principales resultados de este estudio sugieren que al valorar el progreso de los estudiantes en la habilidad para formular preguntas propiciada por el uso de la modelación computacional con diagrama AVM, se encuentra que los tres estudiantes llegaron a un nivel medio; lo que puede ser considerado bueno, si se parte del hecho de que, de acuerdo con la escala cualitativa definida para valorar el nivel de los estudiantes en la formulación de preguntas, todos se encontraban al inicio de la intervención en un nivel bajo en su capacidad para formular preguntas de interés sobre situaciones problema referidas al campo conceptual de la dinámica Newtoniana; formulando preguntas que generaban respuestas de tipo numérico y respuestas del tipo sí o no; y que difícilmente contribuían a la comprensión de los sistemas físicos estudiados.

Aunque al final de la intervención se siguen evidenciando algunas dificultades en la capacidad de formular preguntas, entre las que sobresale el predominio de preguntas que generan respuestas del tipo sí o no; también es posible notar que algunas de las preguntas formuladas indagan más por relaciones entre conceptos -en comparación con las preguntas formuladas al inicio de la intervención-, exigiendo una mayor comprensión de los conceptos involucrados en el análisis de una determinada situación problema.

\section{Consideraciones finales}

Los resultados de este estudio son considerablemente satisfactorios; más aún si partimos del hecho de que estos estudiantes durante su formación nunca habían sido enfrentados al uso de actividades computacionales y mucho menos al uso de una herramienta como el diagrama AVM. Pero sin lugar a dudas, asumir la tarea de ser ellos quienes -en el proceso de enseñanza-aprendizaje- formulaban las preguntas y no el profesor, trajo consigo todo tipo de reacciones, que les llevó incluso a manifestar inicialmente su incapacidad para llevar a cabo dicha tarea.

Y justamente son estos eventos los que nos llevan a otorgarle un gran valor a la actividad de formulación de preguntas propiciada por la modelación computacional y el uso del diagrama AVM, favoreciendo en los estudiantes un progreso significativo en la habilidad para formular preguntas de interés sobre un determinado campo de conocimiento. Del mismo modo, este tipo de actividad nos permite valorar indicios de un aprendizaje significativo crítico, que puede evidenciarse en la capacidad lograda por los estudiantes para formular preguntas cada vez más relevantes, apropiadas y sustantivas, de manera sistemática sobre los fenómenos físicos abordados. 
La formulación de preguntas en el aula ...

Podría decirse que la habilidad que tiene un estudiante para formular preguntas relevantes y de manera sistemática sobre un campo conceptual, tiene una estrecha relación con los conocimientos previos que éste tiene acerca de dicho campo; por lo que indudablemente, enfrentar a los estudiantes a la formulación de preguntas en el aula de clase se convierte en una valiosa estrategia para favorecer el aprendizaje significativo de un determinado campo de conocimiento, así como para comprender que las preguntas se constituyen en la base sobre la cual se construye el conocimiento científico. Lo que sugiere que es posible abordar la enseñanza de diversos campos de conocimiento a partir del principio del cuestionamiento; y en particular, en coherencia con los fundamentos de la Teoría del Aprendizaje Significativo Crítico. Para futuros trabajos se vislumbra la posibilidad de abordar tópicos relativos a la Física moderna, tanto desde la perspectiva del principio del cuestionamiento, así como desde los demás

principios referentes a aspectos disciplinares, epistemológicos y didácticos, que se enmarcan en esta teoría.

\section{Referencias}

AGUIAR, C. E.; RUBINI, G. A aerodinâmica da bola de futebol. Revista Brasileira de Ensino de Física, São Paulo, v. 26, n. 4, p. 297-306, 2004.

ARAUJO, I. S. Simulacão e modelagem computacionais como recursos auxiliares no ensino de física geral. 2005. 229 f. Tese (Doutorado em Ciencias) - Instituto de Física, Universidade Federal do Rio Grande do Sul, Porto Alegre, 2005.

ARAUJO, I. S.; VEIT, E. A.; MOREIRA, M. A. Um estudo exploratório sobre as potencialidades do diagrama AVM na aprendizagem significativa de tópicos de física. Indivisa: boletín de estudios e investigación, Madrid, v. 1, p. 503-514, 2007.

ARAUJO, I. S.; VEIT, E. A.; MOREIRA, M. A. Modelos computacionais no ensinoaprendizagem de Física: um referencial de trabalho. Investigações em Ensino de Ciências, Porto Alegre, v. 17, n. 2, p. 341-366, 2012.

ARNAL, J.; DEL RINCÓN, D.; LATORRE, A. Investigación educativa: fundamentos y metodologías. Barcelona: Labor, 1992.

AUSUBEL, D. P. Adquisición y retención del conocimiento: una perspectiva cognitiva. Barcelona: Paidós Ibérica, 2002.

BACHELARD, G. La formación del espíritu científico. México: Siglo XXI Editores, 1982.

BOBBY, Z. et al. Formulation of questions followed by small group discussion as a revision exercise at the end of a teaching module in biochemistry. Biochemistry and Molecular Biology Education, New York, v. 35, n. 1, p. 45-48, 2007.

BUNGE, M. Teoría y Realidad. 1. ed. Barcelona: Ariel, 1972. 
López, S.; Veit, E. A.; Araujo, I. S.

COLLINS, A.; BROWN, J. S.; NEWMAN, S. E. Cognitive apprenticeship: teaching the crafts of reading, writing, and mathematics. In: RESNICK, L. Knowing, learning, and instruction: essays in honor of Robert Glaser. Hillsdale: Erlbaum, 1990. p. 453-494.

DILLON, J. T. The remedial status of student questioning. Journal of Curriculum Studies, Basingstoke, v. 20, n. 3, p. 197-210, 1988.

GIERE, R. Explaining science: a cognitive approach. Chicago: University of Chicago, 1988.

HALLOUN, I. Schematic modeling for meaningful learning of physics. Journal of Research in Science Teaching, New York, v. 33, n. 9, p. 1019-1041, 1996.

Modeling theory in science education. Boston: Kluwer Academic, 2004.

HESTENES, D. Modeling software for learning and doing physics. In: BERNARDINI, C; TARSITANI, C.; VINCENTINI, M. (Org.). Thinking physics for teaching. New York: Plenum, 1995. p. 25-66.

KING, A. Autonomy and question asking: the role of personal control in guided studentgenerated questioning. Learning and Individual Differences, New Haven, v. 6, n. 2. p. 163-185, 1994.

MOREIRA, M. A. Aprendizaje significativo: teoría y práctica. Madrid: Visor, 2000. 2005.

Aprendizaje significativo crítico. Porto Alegre: Instituto de Física, UFRGS,

NEBER, H.; ANTON, M. Promoting pre-experimental activities in high-school chemistry: focusing on the role of students' epistemic questions. International Journal of Science Education, London, v. 30, n. 13, p. 1801-1821, 2008.

OTERO, J.; GRAESSER, A. PREG: elements of a model of question asking. Cognition and Instruction, Mahwah, v. 19, n. 2, p. 143-175, 2001.

PÉREZ-SERRANO, G. P. Investigación cualitativa: retos e interrogantes. Madrid: La Muralla, 1998.

POSTMAN, N.; WEINGARTNER, C. Teaching as a subversive activity. New York: Dell Publishing Company, 1969.

ROSENSHINE, B.; MEISTER, C.; CHAPMAN, S. Teaching students to generate questions: a review of intervention studies. Review of Educational Research, Washington, v. 66, n. 2, p. 181-221, 1996.

SINGER, H. Active comprehension: from answering to asking questions. Reading Teacher, Newark, v. 31, n. 8, p. 901-908, 1978.

STAKE, R.E. Investigación con estudio de casos. Madrid: Morata, 1998.

Artigo recebido em 06/06/13. Aceito em 02/10/13. 